Guest Editors: Jose C. Nicolau, Claudia F Gravina and Roberto A. Franken

\title{
Heart failure in the elderly
}

\author{
Elizabete Viana de Freitas ${ }^{1,2}$, Michel Batlouni ${ }^{3,4,5}$, Roberto Gamarsky ${ }^{2,6}$ \\ ${ }^{1}$ Department of Cardiology, Pedro Ernesto University Hospital, State University of Rio de Janeiro, Boulevard 28 Setembro, 77 Vila Isabel, Brazil \\ ${ }^{2}$ Department of Geriatrics, Pró-Cardíaco Hospital, Rua General Polidoro 182, Brazil \\ ${ }^{3}$ Department of Cardiology, Avenida Doutor Arnaldo, 455, Consolação, Sao Paulo, CEP: 01246-000, Brazil \\ ${ }^{4}$ Department of Internal Medicine, Federal University of Goiás, Av. Contorno s/nº. Estado: GO Cidade: Gioiânia Bairro: Central CEP: 74055-140, Brazil \\ ${ }^{5}$ Department of Cardiology, Dante Pazzanese Institute, Av. Doutor Dante Pazzanese, $n^{\circ}$ 500. Bairro: Ibirapuera/Vila Mariana Cidade: São Paulo, Brazil \\ ${ }^{6}$ Department of Geriatrics, Federal University of Rio de Janeiro, RJ 20551-030, Brazil
}

\begin{abstract}
The aging of the population is, currently, a major phenomenon, drawing the attention of a number of investigators. The significant increase of life expectancies over the past few decades, in addition to social and economic consequences, has lead to a major change in the morbidity and mortality profile of elders. Heart failure (HF) is a condition in which the heart can not pump enough blood to meet the body's needs. HF is predominantly a disorder of the elderly with rates increasing exponentially. The prevalence of HF approximately doubles with each decade of life. As people live longer, the occurrence of HF rises, as well as other conditions that complicate its treatment. Impaired heart function implies a reduced duration of survival. Fortunately, many factors that can prevent HF and improve outcome are known and can be applied at any stage. This review emphasizes the importance of factors inherent in aging itself, focusing on heart disease, particularly as a disease of aging, can help critically refine management of this acute and chronic disease, as well as foster preventive strategies to reduce the incidence of this common malady.
\end{abstract}

J Geriatr Cardiol 2012; 9: 101-107. doi: 10.3724/SP.J.1263.2011.12295

Keywords: Elderly people; Geriatric problems; Heart failure; Hypertension

\section{Heart failure}

Heart failure (HF) is a complex clinical syndrome resulting from the inability of the heart to adequately supply the metabolic demands of tissues, or do so only with elevated filling pressures. HF can result from disturbance of contractility, with reduced ejection fraction of the left ventricle (systolic HF) or by alterations in the filling and/or ventricular relaxation, with a preserved ejection fraction (diastolic $\mathrm{HF}$ or HF with preserved systolic function).

$\mathrm{HF}$ is a disease of high prevalence and incidence throughout the world. About 400,000 new cases are diagnosed in the United States every year. Data from the Framinghan study demonstrate that the incidence of HF increases progressively in both genders according to age. ${ }^{[1]}$ This prevalence is about

Correspondence to: Elizabete Viana de Freitas, PhD, Department of Cardiology, Pedro Ernesto University Hospital, State University of Rio de Janeiro, Rua Almte, Benjamim Sodré 40/702 Laranjeiras Rio de Janeiro RJ, CEP 22240-080, Brazil. E-mail: Elizabet.rlk@terra.com.br

Telephone: $+55-21-25537288$

Fax: $+55-21-25536999$

Received: December 29, 2011

Revised: April 11, 2012

Accepted: April 18, 2012

Published online: June 1, 2012
$3.3 \%$ in population $\leq 45$ years, $10.5 \%$ in individuals $\geq 65$ years, and around $20.0 \%$ in population $\geq 75$ years $^{[2,3]}$ making HF is the leading cause of hospitalization in the geriatric population.

The most common causes of HF in the elderly are coronary atherosclerotic disease and arterial hypertension, which often coexist. Other common causes in developing countries are: arrhythmias, endocrinopathies, infiltrative, idiopathic and dilated cardiomyopathy, infection and alcohol. ${ }^{[4]}$ Age is also an isolated risk factor for the development of HF after acute myocardial infarction.

Clinically, HF is manifested by physical exercise intolerance, fluid retention, edema and visceral congestion, which often leads to hospitalization, poor quality of life and reduced life expectancy. In recent decades, HF has become increasingly prevalent phenomenon closely linked to the aging population, with growing awareness in clinical geriatrics.

\section{Clinical diagnosis}

The careful analysis of signs and symptoms is crucial for establishing the diagnosis; however, in the elderly, it is

http://www.jgc301.com; jgc@jgc301.com | Journal of Geriatric Cardiology 
difficult to interpret, due to the concomitance of other diseases and by the atypical way they are externalized. ${ }^{[5]}$ The evaluation should be initiated by a well-conducted anamnesis followed by a thorough physical examination and appropriate laboratory tests.

The manifestations of HF can be variable, depending on the time course of the syndrome and the possibility of activation of compensatory mechanisms, ranging from light asymptomatic ventricular dysfunction to acute left ventricular failure.

In elderly patients, the complaint of fatigue is quite common and should not be considered a symptom of aging itself. It is a rather complex symptom related to low cardiac output, peripheral hypo-perfusion and skeletal muscle deconditioning, which may lead to misdiagnosis. ${ }^{[6]}$

The exertional dyspnea, orthopnea, lower extremity edema and reduced exercise tolerance are the cardinal symptoms of $\mathrm{HF}$, in both the young and the elderly, but it can be difficult to interpret, especially in the elderly, in the obese and in women. ${ }^{[2]}$

Dyspnea is the primary symptom of $\mathrm{HF}$, with a progressive and varied intensity according to the condition of cardiac performance.

The initial presentation is exertional dyspnea. In the elderly, it may be associated with increasingly sedentary lives. Dyspnea progresses to orthopnea, paroxymal nocturnal dyspnea, dyspnea at rest and, without any intervention, leads to acute pulmonary edema. Dyspnea is the clinical expression of pulmonary venocapilar hypertension, but fatigue and tiredness are related to lower perfusion and vasoconstriction of skeletal muscle.

In contrast, atypical symptoms, such as confusion, memory deficit, sleepiness, episodes of delirium, irritability, syncopal states, fatigue, anorexia, and reduced level of activity, gradually become common manifestations of HF in the elderly, especially after age 80 . In the geriatric population, gastrointestinal symptoms, such as nausea, vomiting, constipation or diarrhea, occur more often and when associated with anorexia will lead to cachexia. Once the diagnosis has been established, it is recommended to scale the severity of $\mathrm{HF}$ through the symptoms (Table 1).

Table 1. Heart failure functional classification (New York Heart Association).

Class I - No difficulty with usual physical activities, with no manifestation of dyspnea, fatigue or palpitations

Class II-Slight limitation for usual physical activities. Patient asymptomatic at rest; in physical activity, expression of fatigue, dyspnea and palpitations.

Class III-Significant limitation of physical activities, although comfortable at rest. Symptoms of dyspnea, fatigue and palpitations on exertion.

Class IV-Symptoms present even at rest, and discomfort with any physical activity.
The diagnosis of HF in the elderly may be difficult due to the presence of co-morbidities, with increasing prevalence of atypical symptoms and signs. Usually, the physical examination shows clinical signs that vary with the extent and severity of the disease. In the early stages, the patient may experience relatively good clinical status, while chronically ill patients may be malnourished or cachexia. ${ }^{[7,8]}$ Edema due to increased hydrostatic pressure and sodium retention is often found in the elderly, requiring special attention. However, it is often underrated as a sign of HF, for it may be due to the clinical picture of malnutrition with hypo-proteinemia, venous circulatory disorder or renal origin. Peripheral edema is preceded by hepatomegaly, which may progress to anasarca, with ascites and pleural effusion. Pleural effusion, when unilateral, is generally prevalent in the right hemi-thorax.

Fine rales at the lung bases in elderly lose fidelity, not an uncommon complication, considering that in this age group the lack of physical activity or periods of prolonged bed rest lead to the appearance of rales in the bases.

The great variability in the detection and interpretation of signs and symptoms by the examiners, however, are associated with low sensitivity and specificity in clinical diagnosis of HF in the elderly, leading some authors to develop criteria in order to improve the diagnostic accuracy. ${ }^{[9,10]}$ The Framingham researchers listed criteria classified into major and minor from the signs and symptoms of HF, which were used in this study. The presence of two major signs (main criteria) or one major and two minor signs (secondary criteria) would be needed for the diagnosis of HF (Table 2).

The HF may be caused by either systolic or diastolic dysfunction, or the combination of both. ${ }^{[11]}$ The differential diagnosis of these clinical entities, with the determination of the predominance of one over the other, is essential for successful treatment. In $50 \%$ of the patients, particularly those at 70 years old or more, the HF is caused by alterations in the diastolic function. When HF is caused by isolated diastolic dysfunction, congestive symptoms result from pulmonary venous hypertension, with normal systolic function and without

Table 2. Clinical criteria for the diagnosis of heart failure. ${ }^{[1]}$

\begin{tabular}{ll}
\hline Major criteria & Minor criteria \\
\hline Paroxynal nocturnal dyspnea & Ankle edema \\
Jugular venous distention & Coughing at night \\
Hepato jugular reflection & $\begin{array}{l}\text { Hepatomegaly } \\
\text { Pleural effusion }\end{array}$ \\
Rales & Vital capacity less than or equal to the \\
Acute pulmonary edema & one third of the predicted maximum \\
Cardiomegaly & Tachycardia 120 beats/minutes \\
Third heart sound (gallop) & \\
$\geq 4.5$ kg weight loss in 5 days in & \\
response to the treatment of Heart \\
failure.
\end{tabular}


the occurrence of increased cardiac area. ${ }^{[12]}$ The mortality rate in these cases is comparable to that of systolic HF, with approximately $50 \%$ survival rate at five years. The diagnosis of HF should, in any situation, seek to identify the underlying cause, a fundamental condition for the specific and individualized therapy.

\section{Etiology and precipitating factors}

Arterial hypertension and coronary artery disease account for $70 \%$ to $80 \%$ of cases of HF in the elderly. ${ }^{[13,14]}$ The main root causes of $\mathrm{HF}$ are displayed in Table 3 .

Table 3. Common causes of heart failure.

\begin{tabular}{l}
\hline Coronary artery disease: acute and chronic \\
\hline Hypertensive heart disease \\
Valvular heart disease \\
Cardiomyopathy \\
Ischemic \\
Non-ischemic: alcohol; chemotherapy; inflammatory myocarditis; \\
idiopathic dilated cardiomyopathy \\
Hypertrophic \\
Obstructive \\
$\quad$ Unobstructive \\
Restrictive \\
Pericardial disease \\
Triggering factors \\
Chronic anemia; thiamine deficiency; hyperthyroidism; arteriovenous \\
fistula; fever; medications; diet high in salt and water; endocrine \\
diseases; chronic obstructive pulmonary disease; non adherence to \\
therapy; arrhythmias; renal failure; pulmonary embolism. \\
Diastolic dysfunction related to age
\end{tabular}

\section{Complementary investigations}

Complementary examinations aid in the diagnosis of HF, and contribute to the identification of the causative or precipitating factors. The electrocardiogram, although not specific, is usually abnormal, showing a negative predictive value of about $90 \%{ }^{[15]}$ It can display rhythm disturbances, bundle branch blocks, cardiac chamber overload as well as signs of myocardial ischemia. According to the II Cardiogeriatrics Guidelines (2010), (grade of recommendation I, level of evidence. ${ }^{[9,16]}$

Chest radiography (CR) should be part of the initial investigation of HF. A careful evaluation is required for the elderly. Some symptoms of HF may be confused with chronic obstructive pulmonary disease (COPD) and respiratory infections. The finding of cardiomegaly favors the diagnosis of HF, especially if associated with pulmonary congestion and pulmonary hypertension with reversal of the vascular pattern, presence of Kerley lines and pleural effusions. In the elderly, chest deformities, a feature of the aging process, make difficult the interpretation of the cardiac area. CR is the most common diagnostic method in clinical practice (grade of recommendation I and level of evidence C). ${ }^{[15]}$

Doppler echocardiography should also be performed routinely. It is indispensable for the accurate diagnosis of HF because it provides data to the anatomical and functional assessment. It is crucial for the diagnosis of heart diseases through its capacity to quantify valvular lesions, pressure gradients, diameter of cardiac cavities, wall thickness, myocardial contractility, ventricular ejection fraction and ventricular complaisance and relaxation.

Ejection fraction below $45 \%$ corroborates the diagnosis of ventricular dysfunction. On the other hand, ejection fraction $\geq$ $45 \%$, with signs and/or symptoms of HF and echocardiographic abnormalities consistent with alterations in left ventricular filling characterize left ventricular dysfunction with preserved ejection fraction (Grade of recommendation I and level of evidence B). ${ }^{[9,15]}$

The exercise test (ET) has limited value for diagnosis of $\mathrm{HF} \cdot{ }^{[15]} \mathrm{A}$ maximum normal test in patients, without treatment, rules out the diagnosis. On the other hand, in patients on drug therapy, the analysis of parameters obtained in the ET may have limitations due to resulting pharmacological effects. However, ET is useful in assessing the functional capacity and patient's response to the treatment. ${ }^{[15]}$

The 6-minute-walk test is a good choice for the functional evaluation of patients with HF. It is better tolerated than the ET, inexpensive, and can provide important information, both prognostic and therapeutic assessment, in rehabilitation programs. The distances below 300 meters are of poor prognosis, while the ones at 450 meters are correlated with lower rates of mortality and hospitalization. The radionuclide ventriculography is performed to assess ventricular ejection fraction with higher accuracy compared to echocardiography. Its disadvantages are the high cost, time to perform it and exposure to radiation (grade of recommendation level of evidence A). ${ }^{[9]}$

The $\beta$-type natriuretic peptide (BNP) is a hormone produced mainly by ventricular cardiomyocytes, whose secretion is associated with the stretching of myocardial fibers. Quantitative analysis of plasma concentrations of BNP are useful to confirm the diagnosis, provide an estimate prognosis and guide treatment in patients with HF. Elevated plasma concentrations of BNP have $97 \%$ sensitivity and a specificity of $84 \%$ for the diagnosis of HF due to systolic dysfunction, with a negative predictive value around $98 \%$. The low specificity limits its diagnostic value in the diastolic dysfunction. ${ }^{[17]}$

However, such concentrations can establish the differential diagnosis between diastolic HF dyspnea and non-cardiac disease assessments in emergency conditions (grade of reco- 
mmendation I level of evidence A). ${ }^{[9]}$ It is particularly indicated when given access to echocardiography is limited.

Laboratory tests are performed in order to identify associated diseases and to evaluate blood glucose, electrolyte disturbances and renal function. Other tests should be performed with specific clinical indications, such as evaluation of thyroid, respiratory and liver function.

\section{Treatment}

The treatment of HF is scheduled in Table 4.

Table 4. Treatment of heart failure. ${ }^{[15,17]}$

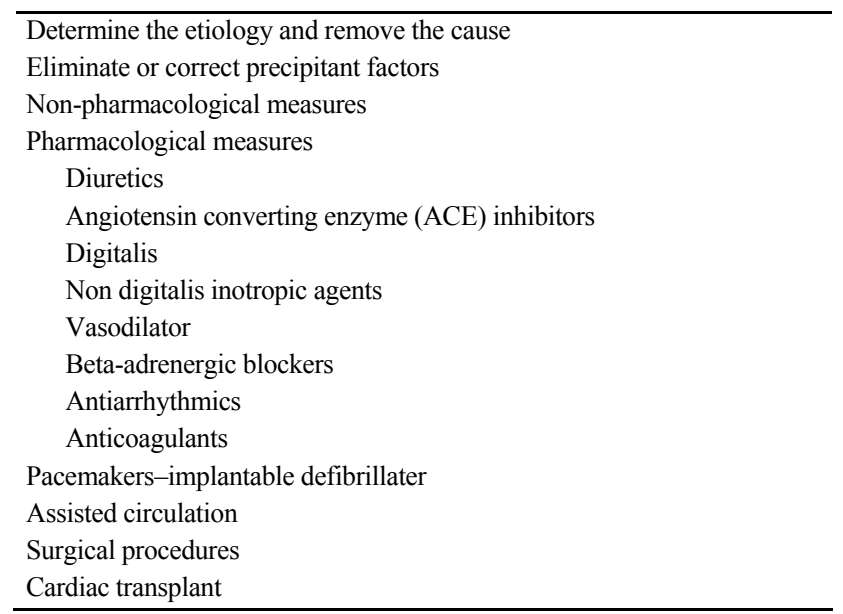

\subsection{Non-pharmacological measures}

Non-pharmacologic measures include appropriate diet, cessation of smoking, increased physical activity, and immunization. ${ }^{[9,15,17]}$ The measures also include dietary sodium restriction, alcohol and net weight reduction in obese patients and nutritional care in patients with cachexia, an important predictor of longer life expectancy. ${ }^{[2]}$ Elderly patients with HF should be immunized against influenza and pneumococcus, although there is no documented evidence of effectiveness. Patients should stay home uncompensated. ${ }^{[15,17]}$ The stable, functional class II-III, should perform physical activity through well planned physical conditioning programs resulting in an increased tolerance to stress. Prolonged bed rest, wherever possible, should be contraindicated. In addition, the use of certain medications should be avoided, including anti-inflammatory steroids, tricyclic antidepressants, corticosteroids, lithium, class I antiarrhythmic agents, among others. ${ }^{[15]}$

\subsection{Pharmacotherapy}

The treatment of HF in the elderly is similar to that of younger cohorts, having to respect some peculiarities of this age group. The normal doses of drugs are, in general, less well tolerated and it is necessary to be careful with the maximum doses recommended in clinical studies. Co-morbidities should be considered, as well as poly-pharmacy, common in the elderly who are most likely prone to iatrogenic and drug interactions.

\subsubsection{Diuretics}

These medications act more quickly in controlling symptoms of HF when fluid retention is present in the form of edema and pulmonary congestion. Their use results in rapid control of dyspnea and improves physical exercise tolerance. ${ }^{[9,15,17]}$ Loop diuretics are initially used (grade of recommendation I, level of evidence C). Thiazide diuretics should be added when there is refractoriness to loop diuretics. Thiazides, however, lose their efficacy in patients with moderate renal impairment (creatinine clearance $\leq 30 \mathrm{~mL} /$ minutes).

Among the potassium sparing diuretics, spironolactone was associated with a $27 \%$ reduction in total mortality. It should be considered in patients in functional class III-IV and should be accompanied by strict control of potassium and creatinine. In the elderly, it is recommended to avoid the association with ACE inhibitors, angiotensin receptor blockers (ARBs) and aldosterone inhibitors. ${ }^{[18]}$

The elderly are more prone to adverse reactions, therefore the treatment should be started with low doses. The main diuretics and their dosages are listed in Table 5.

Table 5. Oral diuretics.

\begin{tabular}{|c|c|c|c|c|c|}
\hline & \multicolumn{2}{|c|}{ Initial dose (mg) } & \multicolumn{2}{|c|}{ Maximum dose daily (mg) } & Side effects \\
\hline \multicolumn{6}{|l|}{ Loop diuretic } \\
\hline Furosemide & \multicolumn{2}{|c|}{$20-40$} & \multicolumn{2}{|c|}{$250-500$} & Hypocalemia, hypomagnesemia, \\
\hline \multirow{2}{*}{ Bumetamide } & \multirow{2}{*}{\multicolumn{2}{|c|}{$0.5-1.0$}} & \multirow{2}{*}{\multicolumn{2}{|c|}{$5-10$}} & hyponatremia \\
\hline & & & & & $\begin{array}{l}\text { Hyperuricemia, intolerance glucose, } \\
\text { Basic acid disturbance }\end{array}$ \\
\hline \multicolumn{6}{|l|}{ Thiazide } \\
\hline Hydrochlorothiazide & \multicolumn{2}{|c|}{25} & \multicolumn{2}{|c|}{100} & $\begin{array}{l}\text { Hypocalemia; hypomagnesemia, } \\
\text { hyponatremia }\end{array}$ \\
\hline Indapamine & \multicolumn{2}{|c|}{2.5} & \multicolumn{2}{|c|}{2.5} & Basic acid disturbance \\
\hline Potassium sparing & $+\mathrm{ACEi}$ & - ACEi & $+\mathrm{ACEi}$ & - ACEi & \\
\hline Amiloride & 2.5 & 5 & 20 & 40 & Hypercalemia, rush \\
\hline Triamterene & 25 & 50 & 100 & 200 & Hypercalemia \\
\hline Espironolactone & $12.5-25$ & 50 & 50 & $100-200$ & Hypercalemia, gynecomastia \\
\hline
\end{tabular}

ACEi: Angiotensin Converting Enzyme inhibitors. 


\subsubsection{Digitalis}

Some studies show that digitalis related compounds are effective in controlling symptoms and improving physical exercise tolerance in patients with HF. Digoxin is a first line drug for treating atrial fibrillation associated with HF (grade of recommendation IIa, level of evidence A). ${ }^{[19]}$

The elderly are less responsive to the effects of digitalis and experience higher toxic effects due to lower muscle mass, associating itself with the highest myocardial concen tration for the same dose. The serum concentration of digoxin is effective from $0.5 \mathrm{ng} / \mathrm{mL}$ to $0.9 \mathrm{ng} / \mathrm{mL}$, whereas higher concentrations are associated with increased toxicity. Its side effects can be exacerbated by the concomitant use of other cardioactive drugs. The daily dose in the elderly should not exceed $0.125 \mathrm{mg}$ and the plasma concentration should not exceed $1 \mathrm{ng} / \mathrm{mL} .^{[20]}$

\subsubsection{ACE inhibitors}

Several studies have shown that ACE inhibitors reduce total mortality determined by HF and the combined events compared to placebo. ${ }^{[15]}$ Despite scientific evidence of improvement of symptoms, reduction of disease progression and reducing mortality and hospitalization for HF, there are limited studies for patients older than 75 years old. ${ }^{[15]}$

ACE inhibitors should be given to all patients with HF and systolic left ventricular dysfunction after myocardial infarction, unless there is contraindication (grade of recommendation is I and level of evidence A). ${ }^{[9]}$ But in patients with diastolic dysfunction the value of ACE inhibitors, per se, has not been established.

ACE inhibitors are contraindicated when serum potassium is above $5.5 \mathrm{mEq} / \mathrm{L}$ in the presence of bilateral renal artery stenosis, symptomatic hypotension, renal failure and previous history of angioedema with their use.

Low doses should be started in the elderly with gradual increases until the recommended doses in clinical trials have been reached. However, more often in the elderly irritating dry cough, hypotension, renal failure and reduction or loss of taste occur, leading to loss of appetite. ${ }^{[21]}$

The main drugs are listed in Table 6.

\subsubsection{ARBs}

ARBs are more appropriate when there is intolerance to ACE inhibitors or in association to these drugs for a limited time. ACE inhibitors remain the agents of choice in the treatment of HF.

The main studies that evaluated the benefit of ARBs in the treatment of HF are VAL-HeFT ${ }^{[22]}$ and CHARM, ${ }^{[23]}$ which included elderly and showed significant results in reducing mortality. ARBs are mainly indicated in patients with chronic $\mathrm{HF}$ and systolic ventricular dysfunction who have intolerance to ACE inhibitors.
Table 6. Angiotensin converting enzyme inhibitors.

\begin{tabular}{lll}
\hline Drug & Initial dose & Drug target dose \\
\hline Captopril & $6.25 \mathrm{mg}, \mathrm{tid}$ & $50 \mathrm{mg}, \mathrm{tid}$ \\
Enalapril & $2.5 \mathrm{mg}, \mathrm{bid}$ & $10 \mathrm{mg}, \mathrm{bid}$ \\
Lisinopril & $2.5-5.0 \mathrm{mg} / \mathrm{d}$ & $20 \mathrm{mg} / \mathrm{d}$ \\
Ramipril & $2.5 \mathrm{mg} / \mathrm{d}$ & $10 \mathrm{mg} / \mathrm{d}$ \\
Perindopril & $2 \mathrm{mg} / \mathrm{d}$ & $8 \mathrm{mg} / \mathrm{d}$ \\
\hline
\end{tabular}

\subsubsection{Beta-blockers}

Clinical studies ${ }^{[24-26]}$ clearly show the benefits of treatment with beta blockers. The SENIORS, ${ }^{[27]}$ a placebo-controlled trial using nebivolol and involving 2128 subjects aged 70 years or more, showed a risk reduction of $14 \%$ for the end point (death and hospital admissions for any cardiac reason). ${ }^{[26]}$

The combination of a beta-blocker and conventional therapy with diuretics, ACE inhibitors and digitalis leads to an improvement of symptoms, functional class and left ventricular function (grade of recommendation I, level of evidence A). The dosage of beta-blockers used in HF are shown in Table 7.

When the target doses are not tolerated, lower doses should be maintained. Beta blockers are contraindicated in the presence of bradycardia, advance atrioventricular block (AVB), hypotension with blood pressure below $90 \mathrm{mmHg}$, bronchospastic disease and in decompensated HF.

Side effects are more common in the elderly, mainly asthenia, fatigue, sleep disturbance, peripheral vascular disorder, bradycardia and AVB.

Table 7. Doses of beta blockers used in heart failure.

\begin{tabular}{lcc}
\hline & Initial dose (mg) & Target dose (mg) \\
\hline Carvedilol & $3.125, \mathrm{bid}$ & $25, \mathrm{bid}$ \\
Sustained-release & $12.5, \mathrm{qd}$ & $200, \mathrm{qd}$ \\
Metopropolol (succinate) & $1.25, \mathrm{qd}$ & $10, \mathrm{qd}$ \\
Bisoprolol & & \\
\hline
\end{tabular}

\subsubsection{Direct vasodilators}

The combination of isosorbide dinitrate and hydralazine for the treatment of HF is recommended for patients on digitalis, diuretics and beta-blockers, that cannot tolerate ACE inhibitors or ARBs, or in presence of renal failure, hyperkalemia $(\mathrm{K}>5.5 \mathrm{meq} / \mathrm{L})$ or worsening of glomerular filtration rate with serum creatinine $>2.5 \mathrm{mg} / \mathrm{dL}$ (grade of recommendation IIa, level of evidence B). ${ }^{[9]}$

\subsubsection{Anticoagulants}

Anticoagulants are indicated in patients with atrial fibrillation, a history of thromboembolic events and left ventricular aneurysm. The use of subcutaneous unfractionated heparin is indicated for patients with decompensated HF and bedridden for the prevention of pulmonary thromboembolism. ${ }^{[9,15]}$ 


\section{Resynchronizers}

Ventricular resynchronization is indicated in patients with advanced HF in functional class III or IV, ejection fraction less than or equal to $35 \%$ and a QRS complex equal to or greater than 0.12 seconds, associated with optimal pharmacological therapy (grade of recommendation I, level of evidence A). ${ }^{[9,28]}$

Resynchronization is not indicated in patients whose functional condition and life expectancy are limited by noncardiac causes (grade of recommendation III, level C). ${ }^{[9]}$

\section{Diastolic heart failure (DHF)}

DHF or HF with preserved systolic function has a high prevalence (about 50\%), involving specially the elderly, women hypertensive, and diabetics patients. Its diagnosis is based on symptoms and signs of HF in patients with normal ejection fraction in the echocardiogram. ${ }^{[11]}$ The inclusion of the BNP measurement can increase the diagnostic accuracy. ${ }^{[2]}$

In contrast with the treatment of systolic heart failure, only few clinical trials are available to guide the management of patients with DHF. It is important, however, to follow some general principles: (1) Control of the heart frequency and arterial blood pressure, (2) Reduction of the volume overload and (3) Relief of myocardial ischemia. Drug therapy involves mainly the use of diuretics. However, excessive volume depletion and arterial hypotension should be carefully avoided in older patients, because they are more sensitive to preload reduction. Calcium channel blockers, ${ }^{[29]}$ beta-blockers, $\mathrm{ACE}$ inhibitors or $\mathrm{ARBs}^{[30]}$ and espironolactone are useful to relief of symptoms and reduce morbidity and mortality. The use of digitalis is not well established, except in the presence of atrial fibrillation with high ventricular response.

\section{References}

1 Ho KK, Pinsky JL, Kannel WB, et al. The epidemiology of heart failure: The Framingham Study. J Am Coll Cardiol 1993; 22(4 Suppl A): 6A-13A.

2 Kannel WB, Ho K, Thom T. Changing epidemiological features of cardiac failure. Br Heart J 1994; 72(2 Suppl): S3-S9.

3 Petrie MC, Berry C, Stewart S, et al. Failing aging hearts. Eur Heart J 2001; 22: 1978-1990.

4 Weir RAP, Mcmurray JJV, Taylor J, et al. Heart failure in older patients. Br J Cardiol 2006; 13: 257-266.

5 Abdelhafz AH. Heart failure in older people: causes, diagnosis and treatment. Age Ageing 2002; 31: 29-36.

6 Gillespie ND. The diagnosis and management of chronic heart failure in older patient. Br Med Bull 2006; 75-76: 49-62.
7 Wenger NK. Cardiovascular disease in the elderly. Curr Probl Cardiol 1992; 17: 611-690.

8 Rich MW. Heart failure: Epidemiology, Pathophysiology and Management. In Cardiovascular Disease in the Octogenarium and Beyond; Wenger NK, Ed; Martin Dunitz: London, UK, 1999; 73-91.

9 Gravina CF, Rosa RF, Franken RA, et al. Guidelines in Cardiogeriatrics II of the Brazilian Society of Cardiology. Arq Bras Cardiol 2010; 95(Supl.2): S1-S112.

10 Franklin SS, Gustin W, Wong ND, et al. Hemodynamic patterns of age-related changes in blood pressure. The Framingham Heart Study. Circulation 1997; 96: 308-315.

11 Paulus Wj, Tschöpe C, Sanderson JE, et al. How to diagnose diastolic heart failure: a consensus statement on the diagnosis of heart failure with normal left ventricular ejection fraction by Heart Failure and Echocardiography Associations of the European Society of Cardiology. Eur Heart J 2007; 28: 2539-2550.

12 Haney S, Sur D, Xu Z. Diastolic heart failure. A review and primary care perspective. J Am Board Fam Med 2005; 18: 189-198.

13 Batlouni M, Freitas EV, Neto Savioli F. Heart Failure in the Elderly. In Treaty of Geriatrics and Gerontology; $3^{\text {rd }}$ Edition; Freitas EV, Py L, Eds.; Rio de Janeiro: Brazil 2011; 462-483.

14 Batlouni M, Savioli Neto F, Magalhães HM. Alterações Farmacocinéticas e Farmacodinâmicas Relacionadas à Idade. Terapêutica Cardiovascular no Idoso. In Farmacologia $e$ Terapêutica Cardiovascular; Batlouni M, Ramires JAF, Eds.; São Paulo: Atheneu 1999: 27-46.

15 Swedberg K, Cleland J, Dargie H, et al. Guidelines for the diagnosis and treatment of chronic heart failure: executive summary (update 2005): the task force for the diagnosis and treatment of chronic heart failure of the European Society of Cardiology. Eur Heart J 2005; 26: 1115-1140.

16 Davie AP, Francis CM, Love MP, et al. Value of the electrocardiogram in identifying heart failure due to left ventricular systolic dysfunction. Br Med J 1996; 312: 222-227.

17 A Report of the American College of Cardiology/American Heart Association Task Force on Practice Guidelines (Writing Committee to Update the 2001 Guidelines for the Evaluation and Management of Heart Failure). Circulation 2005; 112: 1825-1852.

18 The RALES Investigators. Effectiveness of spironolactone added to an angiotensin-converting enzyme inhibitor and a loop diuretic for severe chronic congestive heart failure (The Randomized Aldactone Evaluation Study-RALES). Am $J$ Cardiol 1996; 78: 902-907.

19 Goldsmith S, Marx S. Update use of digitalis and nitrates in the elderly. Geriatrics 1988: 43: 71-76.

20 The effect of digoxin on mortality and morbidity in patients 
with heart failure. The Digitails Investigation Group. $N$ Engl $J$ Med 1997; 336: 525-533.

21 Schwartz S, Zipes DP. Cardiovascular disease in special populations. In Treaty of Cardiovascular Disease; Zipes DP, Libby P, Bonow R, et al., Eds; Saunders: New York, 2006; 1925-1949.

22 Cohn JN, Archibald DG, Ziesche S, et al. Effect of vasodilator therapy on mortality in chronic congestive heart failure. Results of a Veterans Administration Cooperative Study. $N$ Engl J Med 1986; 314: 1547-1552.

23 McMurray JJ, Young JB, Dunlap ME, et al. CHARM Investigators. Relationship of dose of background angiotensinconverting enzyme inhibitor to the benefits of candesartan in the Candesartan in heart failure: Assessment of Reduction in Mortality and morbidity (CHARM)-Added trial. Am Heart $J$ 2006; 151: 985-991.

24 Merit-HF Study Group. Effect of metoprolol CR/XL in chronic heart failure: Metoprolol CR/XL randomized intervention trial in congestive heart failure (MERIT-HF). Lancet 1999; 94: 2807-2816.

25 Packer M, Coats AJS, Fowler MB, et al. Effect of carvedilol in severe chronic heart failure. New Engl J Med 1996; 334; 1349-1355.

26 CIBIS II Investigators and Committies. The Cardiac insufficiency Bisoprol Study: A randodomized trial. Lancet 1999; 353: 9-13.

27 Flather MD, Shibata MC, Coats AJ, et al. Randomized trial to determine the effect of nebivolol on mortality and cardiovascular hospital admission in elderly patients with heart failure (SENIORS). Eur Heart J 2005; 26: 215-225.

28 Leclercq C, Kass DA. Retiming the failing heart: principles and current clinical status of cardiac resynchronization. $J$ Am Coll Cardiol 2002; 39: 194.

29 Setaro JF, Zaret BL, Schulman DS, et al. Usefulness of verapamil for congestive heart failure associated with abnormal left ventricular diastolic filling and normal left ventricular systolic performance. Am J Cardiol 1990; 66: 981-986.

30 Warner JG Jr., Metzger DC, Kitzman DW, et al. Losartan improves exercise tolerance in patients with diastolic dysfunction and a hypertensive response to exercise. J Am Coll Cardiol 1999; 33: 1567-1572. 